\title{
Online von- und miteinander lernen
}

\author{
COPILOT-Projekt Nur eine kleine Zahl der Ergotherapie-Studierenden hat die Möglichkeit, \\ einen Teil des Studiums im Ausland zu absolvieren und dadurch internationale sowie inter- \\ kulturelle Erfahrungen zu sammeln. Um auch ihnen einen Austausch anzubieten, wurde das \\ COPILOT-Projekt ins Leben gerufen, das ein digitales Vernetzen in Europa ermöglicht.
}

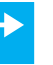

Im Zeitalter der Internationalisierung, Globalisierung und Digitalisierung spielt die akademische Hochschulbildung zur Lösung gesellschaftlicher Herausforderungen eine bedeutende Rolle, um die Zukunft Europas zu gestalten. Das Bevölkerungswachstum verbunden mit globalem und digitalem Fortschritt machen deutlich, dass eine weltumfassende Denkweise und die Fähigkeit, in einem multikulturellen Umfeld effektiv zu agieren, von entscheidender Bedeutung sein werden [1]. Diese Entwicklungstrends fordern neue Kompetenzen, beispielsweise die kritische Reflexion, Kommunikationsfähigkeit, Ressourcennutzung und die digitale Kompetenz [2].

Um diese Fähigkeiten zu entwickeln, nutzen viele Studierende die Möglichkeit, Erfahrungen im Ausland zu sammeln. Allerdings ist dies zum Beispiel aus finanziellen Gründen nicht für alle möglich. Daher wurde das „Cooperative Online Peer-Assisted Intercultural Learning in Occupational Therapy

\section{Hochschulen}

\section{Teilnehmende}

\section{Einrichtungen}

$\rightarrow$ FH Joanneum, Österreich

$\rightarrow$ HOWEST, Belgien

$\rightarrow$ Universität Angel Kanchev, Bulgarien

$\rightarrow$ Universität Lulea, Schweden

$\rightarrow$ Zuyd Hogeschool, Niederlande

$\rightarrow$ ZHAW, Schweiz
(COPILOT)"-Projekt ins Leben gerufen. Es umfasst neun Onlinemodule, die von sechs europäischen Hochschulen (o HOCHSCHULEN) und dem European Network of Occupational Therapy in Higher Education (ENOTHE) entwickelt wurden. Das Projekt wurde durch Erasmus+ Strategische Partnerschaft und die Schweizer Stiftung Movetia gefördert. Eines der Module wird im Folgenden näher vorgestellt.

\section{Modul zur Verbreitung von Ergotherapie $\rightarrow$ Das COPILOT-Modul „OT goes public - Spreading OT in Europe" betrachtet Möglichkeiten zur Ver- \\ 95} breitung von Ergotherapie in Europa. Insgesamt nahmen von September bis November 202037 Studierende aus Belgien, Bulgarien und den Niederlanden (darunter auch Mitautorin Vivienne Weinand) am Modul teil. Dabei arbeiteten sie internationale Marketing-Strategien zur Sensibilisierung für Ergotherapie mit dem Schwerpunkt auf einer selbst gewählten Zielgruppe heraus, zum Beispiel junge Menschen nach einem Schlaganfall.

Als Vorbereitung auf die internationale Zusammenarbeit diente die individuelle Recherche über Ergotherapie im Heimatland. Die Ergebnisse wurden nach dem Kennenlernen innerhalb der Kleingruppen präsentiert und zusammengetragen. Danach identifizierten die Studierenden gemeinsam eine vulnerable Personengruppe als Zielgruppe, die sie in einer SWOT-Analyse genauer beleuchteten (SWOT

steht für Strenghts, Weaknesses, Opportunities und Threats). Ziel der Analyse war das Herausarbeiten von Strategien, wie man die Ergotherapie und ihr Potenzial innerhalb der Zielgruppe öffentlich stärker vertreten kann. Jede und jeder Studierende stellte mindestens zwei Personen dieser Zielgruppe, möglichst auf Mikro-, Makro- und Mesoebene, die Strategien vor und holte sich Feedback ein.

Die Zusammenarbeit fand online über verschiedene Plattformen statt. Die Organisation von gruppeninternen Meetings geschah eigenverantwortlich und ohne festgelegte Zeitfenster im Studienplan der Hochschulen. Zum Ende des Moduls stellten sich die Gruppen gegenseitig ihre Ideen, Ergebnisse und Reflexionen des Prozesses vor. Diese wurden allen Teilnehmenden frei zugänglich zur Verfügung gestellt.

Eine der Kleingruppen beispielsweise wählte Grundschüler*innen mit ADHS als vulnerable Personengruppe aus. Die SWOT-Analyse ergab unter anderem, dass im europäischen Kontext wenig schulbasierte Ergotherapie stattfindet, obwohl diese im internationalen Raum Erfolge zeigt. Die von der Gruppe entwickelten Strategien waren, Flyer mit Informationen über das Potenzial von (schulbasierter) Ergotherapie an Betroffene zu versenden oder eine Onlineplattform zum Austausch von Ergotherapeut*innen, Eltern der betroffenen Kinder und Lehrer*innen über Bedürfnisse, Anforderungen 
etc. zu erstellen. Über die Praktikabilität und Relevanz der Strategien wurden Eltern von einem Jungen mit ADHS (Mikroebene), eine Ergotherapeutin mit einer Fortbildung „Schulbasierte Ergotherapie“ (Mesoebene) und ein angehender Grundschullehrer (Mesoebene) befragt. Konsens des Feedbacks ist, dass Aufklärung sowie Austausch über schulbasierte Ergotherapie als notwendig erachtet werden, da nur geringe Grundkenntnisse vorhanden sind und Bedarf an Unterstützung besteht.

Projekt mit Potenzial $\rightarrow$ COPILOT als Internationalisierungsprojekt ermöglicht Teilnehmenden vielseitige Erfahrungen in der internationalen Vernetzung und dem interkulturellen Lernen, die laut aktuellen Entwicklungstrends an Bedeutung gewinnen. Besonders in der Ergotherapie stellt der internationale Austausch zur Etablierung und zum Empowerment der vergleichbar kleinen Berufsgruppe eine hohe Relevanz dar. COPILOT vermittelt die bedeutsame internationale Best Practice bereits während des Studiums mit dem Ziel, diese anhaltend in den ergotherapeutischen Berufsalltag zu integrieren. In einem globalen Pandemieumfeld ermöglichte das Onlinemodul interkulturelles Lernen unter Bedingungen sozialer Isolation.

Die Teilnahme an einem der COPILOTModule wird Ergotherapie-Studierenden der sechs Kooperationshochschulen jährlich angeboten. Für eine nachhaltige Implementierung des Projekts sind fortlaufende Evaluationen notwendig, um die Qualitätssteigerung in der Lehre und eine Professionsentwicklung weiter voranzutreiben.

Zusammenfassend lässt sich sagen, dass die Teilnehmenden von COPILOT profitieren, da sie vor Herausforderungen gestellt und mit aktuellen Themen konfrontiert werden, die als individuell bedeutsam erlebt werden. Das Outcome ist weitreichend - von der Stärkung der eigenen und der kollektiven Berufsidentität und gemeinsamen Zukunftsvisionen über organisatorischen, kommunikativen sowie individuellen Kompetenzerwerb bis hin zum persönlichen, internationalen Netzwerken.

Vivienne Weinand, Ulla Pott

$\mathbf{a}$ Literaturverzeichnis

www.thieme-connect.de/products/ergopraxis $>$ „Ausgabe $1 / 22$ “

$\overrightarrow{~ L e r n e n ~ a u f ~ v i e l e n ~ E b e n e n ~}$

\section{Erfahrungen aus der Studierendenperspektive}

Die folgenden Berichte umfassen subjektive Erfahrungen, die Studierende im Rahmen des COPILOT-Moduls gesammelt haben. Dabei werden berufsbezogene Aspekte im europäischen Kontext, organisatorische Anforderungen sowie persönlich-interaktionelles Erleben beschrieben.

Berufspolitisch definierten die Studierenden die Ergotherapie alle mit dem zeitgenössischen Paradigma, das sich durch die Kernkonzepte der Betätigungsbasierung und Klientenzentrierung kennzeichnet [3]. Eine weitere Gemeinsamkeit zeigte sich in der Vielfalt von Klient*innen mit Indikation für eine ergotherapeutische Behandlung. Anders war es beim Thema Bildung. Während in Deutschland eine dreijährige schulische Berufsausbildung Standard ist, wurde diese in den anderen Ländern bereits vollständig akademisiert. Zudem unterscheidet sich die Größe des ergotherapeutischen Berufsstandes in den EU-Mitgliedsländern. So gelten in Deutschland die Ergotherapeut*innen mit rund 60000 Angehörigen als zweitgrößte Berufsgruppe der Heilmittelbranche, während in ganz Bulgarien insgesamt nur 50 Ergotherapeut*innen arbeiten. Diese Zahl lässt sich damit erklären, dass die Ergotherapie in Bulgarien erst seit 2006 existiert und die Universität Angel Kanchev die einzige ausbildende Institution des Landes ist.

Organisatorisch gesehen stellte die eigenverantwortliche Arbeit in den Modulen sowohl eine Ressource als auch eine Barriere dar. Zum einen konnten die Studierenden ihre eigenen Bedürfnisse und Lernformen berücksichtigen. Dazu waren Flexibilität, Selbststrukturierung und verbindliche Absprachen gefragt, da sich durch räumliche Distanzen, verschiedene Stundenpläne und private Termine verfügbare Zeitfenster für gemeinsame Meetings unterschieden. Auf der anderen Seite wurde durch den freien Rahmen die Terminvereinbarung erschwert. Dabei spielte die persönliche Motivation für die Gruppenarbeit eine entscheidende Rolle. Aufgrund des kooperativen Miteinanders und Ansprechens von Interessenkonflikten wurden Kompetenzen wie Zeitmanagement, Konfliktmanagement und Kommunikationsfähigkeit erweitert. Letztere stellte durch die Verständigung auf Englisch als Fremdsprache eine Herausforderung dar. Einige Studierende nutzten aufgrund der Partizipation und Praktikabilität digitale Tools, um sprachliche Barrieren zu überwinden, Ideen grafisch zu realisieren oder simultan Dokumente zu bearbeiten.

Persönlich profitieren die Studierenden von COPILOT auf interaktioneller und erlebnisorientierter Ebene sowie bei der Persönlichkeitsentwicklung. Unter anderem bedeutet das Sprechen auf Englisch und das Kennenlernen von Unbekanntem, die eigene Komfortzone zu verlassen. Meistert man diese Herausforderungen, führt das zu erlebter Selbstwirksamkeit und im Kleingruppenkontext zu einem gestärkten Wir-Gefühl. Daraus resultieren Erfolgserlebnisse, die Freude bereiten und auf vielen Ebenen bereichern. Die Auseinandersetzung mit interkultureller Diversität birgt das Potenzial, durch Konfrontation Vorurteile bewusst wahrzunehmen und zu überwinden. Besonders hervorzuheben ist der Kompetenzerwerb während des Austauschprogramms, der zur persönlichen Weiterentwicklung auf beruflicher und privater Ebene führte. Dieser Erwerb schloss sowohl die Aneignung von Fähigkeiten und Fertigkeiten als auch das Erleben eigener Kompetenzen in Interaktion mit anderen ein. Aus der Zusammenarbeit entstanden länderübergreifende Freundschaften, die nach Beendigung des Projekts bestehen blieben.

\section{甶 Autorinnen}

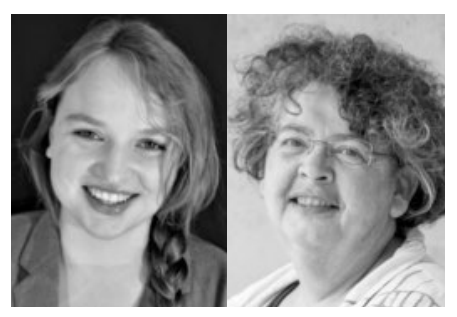

Vivienne Weinand ist seit 2019 Ergotherapeutin und arbeitet in der interdisziplinären Frühförderung der Lebenshilfe Aachen e. V. Im Herbst 2020 nahm sie im Rahmen ihres Bachelorstudiums an dem COPILOT-Projekt mit dem Modul „OT goes public“ teil.

Ulla Pott ist Ergotherapeutin und seit 2003 als Dozentin an der Zuyd Hogeschool in Heerlen (NL) tätig. 